\title{
An ASIP model with general gate opening intervals
}

\author{
Onno Boxma ${ }^{1}$ (D) Offer Kella ${ }^{2}$. Uri Yechiali ${ }^{3}$
}

Received: 18 March 2016 / Revised: 17 June 2016 / Published online: 8 July 2016

(C) The Author(s) 2016. This article is published with open access at Springerlink.com

\begin{abstract}
We consider an asymmetric inclusion process, which can also be viewed as a model of $n$ queues in series. Each queue has a gate behind it, which can be seen as a server. When a gate opens, all customers in the corresponding queue instantaneously move to the next queue and form a cluster with the customers there. When the $n$th gate opens, all customers in the $n$th site leave the system. For the case where the gate openings are determined by a Markov renewal process, and for a quite general arrival process of customers at the various queues during intervals between successive gate openings, we obtain the following results: (i) steady-state distribution of the total number of customers in the first $k$ queues, $k=1, \ldots, n$; (ii) steady-state joint queue length distributions for the two-queue case. In addition to the case that the numbers of
\end{abstract}

Onno Boxma: research done in the framework of the IAP BESTCOM project, funded by the Belgian government and by the Gravity program NETWORKS of the Dutch government.

Offer Kella: supported in part by Grant 1462/13 from the Israel Science Foundation and the Vigevani Chair in Statistics.

$凶$ Onno Boxma

o.j.boxma@tue.nl

Offer Kella

offer.kella@huji.ac.il

Uri Yechiali

uriy@post.tau.ac.il

1 EURANDOM and Department of Mathematics and Computer Science, Eindhoven University of Technology, P.O. Box 513, 5600 MB Eindhoven, The Netherlands

2 Department of Statistics, The Hebrew University of Jerusalem, Mount Scopus, 91905 Jerusalem, Israel

3 Department of Statistics and Operations Research, School of Mathematical Sciences, Tel Aviv University, 69978 Tel Aviv, Israel 
arrivals in successive gate opening intervals are independent, we also obtain explicit results for a two-queue model with renewal arrivals.

Keywords Asymmetric inclusion process - Tandem network - Synchronized service · Queue length distribution

Mathematics Subject Classification 60K25 - 90B22

\section{Introduction}

The asymmetric inclusion process (ASIP), introduced and analyzed in [5-9], is a one-dimensional lattice of $n$ sites (queues), where particles (for example, customers) arrive randomly into the first site ( $\left.Q_{1}\right)$, stay there ('served') for a random time, continue moving simultaneously and unidirectionally from site to site while staying for a random time in each site, until finally exiting the last site $\left(Q_{n}\right)$ and leaving the system. The ASIP defines the missing link between the celebrated Tandem Jackson Network (TJN) and the Asymmetric Exclusion Process (ASEP) [1-3] which plays the role of a paradigm in nonequilibrium statistical mechanics. Imagine that each site has a gate behind it that opens every exponentially distributed random time, allowing particles in the site to move forward to the next site. Denoting by $C_{\text {capacity }}$ the capacity of a site (i.e., maximal number of particles that can reside in the site), and by $C_{\text {gate }}$ the capacity of the site's gate (i.e., the maximal number of particles that can move forward when the gate opens), then, for the TJN, $C_{\text {capacity }}=\infty$ and $C_{\text {gate }}=1$, while for the ASEP $C_{\text {capacity }}=C_{\text {gate }}=1$. When $C_{\text {capacity }}=C_{\text {gate }}=\infty$, one obtains the ASIP model where, when a gate of a site opens, all particles (customers) present there move simultaneously to the next site, joining the particles already there and forming a cluster of particles that continues moving as one unit. In the present work, we generalize the ASIP model by assuming that gate openings are determined by a Markov renewal process such that if, at some time, gate $i$ opens, then with probability $p_{i j}$ the next gate to open is gate $j$, and the time until that gate opens is a random variable $O_{i j}$. We derive the Probability Generating Function (PGF) of the total occupancy (i.e., total number of customers) of sites 1 to $k(k=1,2, \ldots, n)$, while further studying the case when $p_{i j}=q_{j}$. We obtain the joint queue length distribution for the two-queue case, and analyze the system assuming binomial movement of particles. That is, when gate $i$ (say) opens, each particle from the $X_{i}$ particles present in site $i\left(Q_{i}\right)$ will move forward (independently of the other particles) to site $i+1\left(Q_{i+1}\right)$ with probability $a_{i}$, such that the total number of particles moving from $Q_{i}$ to $Q_{i+1}$ is binomially distributed with parameters $X_{i}$ and $a_{i}$.

The ASIP model, first studied in [6], presents a one-dimensional lattice of $n$ queues with Poissonian flow only into the first site. Each gate opens, distinctly and independently of the others, every exponential time with rate $\mu_{k}$ for site $k$, implying that $O_{i j}$ is the same for all $(i, j)$ combinations, and exponentially distributed with rate $\sum \mu_{k}$, while $p_{i j}=\mu_{j} / \sum \mu_{k}, i, j=1,2, \ldots, n$. The multidimensional PGF of the occupancy vector $\left(X_{1}, \ldots, X_{n}\right)$ was studied, and it was shown that this PGF does not exhibit the famous product-form solution characterizing Jackson Networks. Accordingly, an 
iterative solution procedure was developed. However, the PGF of the total-load-upto-site $k, k=1,2, \ldots, n$, was shown to have a product-form solution of geometric variables. For various objective functions, it was shown that the optimal intensities of the gate openings should be equal to each other. Considering large-size ASIP, it was observed in [8], via simulations, that $P\left(X_{k}>0\right) \sim k^{-1 / 2}, E\left[X_{k} \mid X_{k}>0\right] \sim k^{1 / 2}$; and that (standard deviation of $X_{k}$ )/E[ $\left.X_{k}\right] \sim k^{1 / 4}$. Those observations were later proved analytically in [7], where limit laws when $n \rightarrow \infty$ were derived. Various measures were investigated: (i) a particle's (customer's) traversal time, $T$, in a homogeneous ASIP, is distributed as $T \sim n m+n^{1 / 2} m Z$, where $m=$ mean time between successive gate openings, $m^{2}$ is its variance, and $Z$ is the Gaussian $(0,1)$ random variable. (ii) The Laplace-Stieltjes Transform (LST) and mean of the busy period (the time from the first arrival of a customer at an empty system until the first moment thereafter that the network becomes empty again). (iii) The LST and mean of the draining time (the time from an arbitrary moment when the system is in steady state and the inflow is stopped, until the first moment thereafter that the system becomes empty). Occupation probabilities were considered in [9]. Closed-form results were obtained for the probabilities that the total occupation of 'lattice intervals' of $m$ sites, sites $k$ to $k+m-1$, is equal to $l, l=0,1,2, \ldots$ In particular, when $l=0$, the problem becomes a discrete boundary value problem and the probabilities are derived with the aid of Catalan numbers.

The main contribution of this paper is that it considerably extends the exact analysis of ASIP tandem models: We allow the gate openings to be determined by a Markov renewal process, instead of assuming that each gate opens after exponentially distributed intervals, and we extend the Poisson arrival assumption by allowing a quite general arrival process of customers at the various queues during intervals between successive gate openings. Under these assumptions, we determine the steady-state distribution of the total number of customers in the first $k$ queues, $k=1, \ldots, n$. We obtain some additional results for the two-queue case, and solve three optimization problems, thus obtaining insight into the design of ASIPs.

The paper is organized as follows. Section 2 contains the model description. Section 3 is devoted to the analysis of the steady-state joint distribution of the numbers of customers in the various queues just after gate openings. Section 4 contains a brief discussion on optimization of the system performance, for the case that arrivals only occur in $Q_{1}$. A few more detailed two-queue results are presented in Sect. 5. We conclude with some suggestions for further research in Sect. 6.

\section{Model description}

Consider the following model of $n$ queues $Q_{1}, \ldots, Q_{n}$ in series. Each queue has one gate behind it, which may be viewed as a server. Gates are almost all the time closed. When gate $i$ (the gate behind $Q_{i}$ ) opens, all customers present in $Q_{i}$ are instantaneously transferred to $Q_{i+1}, i=1,2, \ldots, n-1$; when gate $n$ opens, all customers present in $Q_{n}$ instantaneously leave the system. After the transfer, the gate immediately closes again. Gate openings are determined by a Markov renewal process. If, at some time $t$, gate $i$ opens, then with probability $p_{i j}$ the next gate to open is gate $j$; and the time until that gate opens is a random variable $O_{i j}$. We assume that the Markov chain governing 
the successive gate openings is irreducible and we denote its steady-state distribution by $\pi_{i}, i=1, \ldots, n$.

During an $O_{i j}$ period, customers may arrive at all queues. We assume that the vectors of arrival numbers in successive gate opening intervals are independent, but may depend on the indices $i$ and $j$. The generating function of the numbers of arrivals into $Q_{1}, \ldots, Q_{n}$ during an $O_{i j}$ period is given by $A_{i j}\left(z_{1}, \ldots, z_{n}\right)$. In addition, we denote the generating function of the cumulative number of arrivals into $Q_{1}, \ldots, Q_{k}$ during an $O_{i j}$ period by $A_{i j k}(z):=A_{i j}(z, \ldots, z, 1, \ldots, 1)$, where the last $z$ occurs at position $k$. Notice that one example is provided by a batch Poisson arrival process, possibly with dependence between batch sizes at different queues, and with arrival rates which may depend on the type of gate opening interval.

The ASIP model as introduced and studied in [5-9] is a generic model that may represent many different stochastic processes in chemistry, physics, and everyday life. From a queueing perspective, it is a series of queues with unlimited batch service. The notion of batch service is closely related to growth-collapse processes. Stochastic growth-collapse temporal patterns appear in a variety of systems, like sandpile models and systems in self-organized criticality, stick-slip models of interfacial friction, Burridge-Knopoff models of earthquakes and continental drift, stochastic avalanche models, and stochastic Ornstein-Uhlenbeck capacitors (cf. page 16 of [5], and references given there). From a statistical physics perspective, the ASIP is a reaction-diffusion model for unidirectional transport with coagulation. Our model significantly generalizes the model of [5-9], allowing us to more accurately represent those stochastic processes. It also allows one to represent movements of ships, crowds, or cars. An ASIP model may represent a series of sluices, with ships simultaneously moving from one section to the next one when a gate is opened. An ASIP may also represent the movement of a crowd through a series of sections of an amusement park-and in both settings it is more natural to model the gate openings by a Markov renewal process than by assuming that all gates open according to independent Poisson processes (independent exponential gate opening intervals). Furthermore, in several settings, for example in a series of road traffic intersections with traffic lights, it is also restrictive to only allow external arrivals at $Q_{1}$. In our model, during a gate opening interval $O_{i j}$, arrivals at all the queues are possible.

We do restrict ourselves to the case in which customers from $Q_{i}$ can only move to $Q_{i+1}, i=1,2, \ldots, n-1$. That assumption will allow us to obtain exact results for the total number of customers $X_{(k)}$ which are present in the first $k$ queues right after a gate opening $(k=1,2, \ldots, n)$. Our results will become somewhat simpler in the special case in which the next gate opening is of gate $j$ with a fixed probability $q_{j}$, i.e., irrespective of the index of the previous gate opening. Notice that the original ASIP model of [5-9] also has this property, as there the gate openings are governed by independent Poisson processes. We work out this special case of fixed gate opening probabilities $q_{j}$ in an example in Sect. 3, showing that just like in $[6,7] X_{(k)}$ can be written as the sum of $k$ independent random variables. We also consider the problem of optimally choosing the gate opening fractions $q_{j}$ in Sect. 4. 


\section{Analysis}

We are interested in the steady-state joint distribution of the numbers of customers $\left(X_{1}, \ldots, X_{n}\right)$ just after a gate opening. To argue the existence of such a distribution, let $\xi_{k}=\left(\xi_{k 1}, \ldots, \xi_{k n}\right)$ be the state of the network right after the $k$ th gate opening and let $g_{k}$ be the gate that opened. Then, because the external arrival process is independent of the process of the gate openings, $\left(\xi_{k}, g_{k}\right)$ is a Markov chain. To argue that it is positive recurrent (on an appropriate state space), let us define an auxiliary process as follows. Let $\eta_{k i}=I\left(\xi_{k i} \geq 1\right)$. Then $\left(\eta_{k}, g_{k}\right)$ is also a Markov chain and $\xi_{k}$ is the zero vector if and only if $\eta_{k}$ is. We note that for every station $j$, the state $(0, j)$ is accessible from every other state. This is because if we block external arrivals, the time until the network becomes empty is finite (actually has a finite expectation). When this happens, we are in some state $(0, \ell)$. Since $g_{k}$ is irreducible, then, if we once again block all arrivals the state $(0, j)$ is accessible from $(0, \ell)$ (actually, without arrivals, it will also be reached after finite expected time). With positive probability, the time until the first arrival is greater than the (independent) time to reach $(0, j)$ without arrivals and thus $(0, j)$ is accessible from any other state. Thus, on the states $(y, j)$ which are accessible from $(0, j)$ (which include $(0, \ell)$ for all $1 \leq \ell \leq n$ and all states that are accessible from $(0, \ell)$ for any such $\ell$ ), we have that $\left(\eta_{k}, g_{k}\right)$ is an irreducible Markov chain and since the state space is finite (contained in or equal to $\{0,1\}^{n} \times\{1, \ldots, n\}$ ) it follows that it is positive recurrent. Therefore, for any $j$, the time between visits to state $(0, j)$ has a finite mean. This implies that the time between visits of $\left(\xi_{k}, g_{k}\right)$ to $(0, j)$ also has a finite mean and thus the $\left(\xi_{k}, g_{k}\right)$ is also positive recurrent on an appropriate state space (all the states which are accessible from $(0, j)$ for some, hence all, $j)$. We note that this idea can be used to argue stability for the continuous time process, which although it is not semi-Markov due to the arrival process, is nevertheless regenerative with finite mean regeneration epochs, provided that $O_{i j}$ have finite means. Since we do not need it here, we omit the details.

In the present section, we shall in particular focus on $X_{(k)}:=X_{1}+\cdots+X_{k}$, viz., the total number of customers in the first $k$ queues right after a gate opening. Introducing $M$, the index of the gate that has just opened, we consider

$$
G_{k i}(z):=\mathbb{E}\left[z^{X_{(k)}} I(M=i)\right], \quad k, i=1, \ldots, n,
$$

where $I(\cdot)$ denotes an indicator function. The fact that customers can only move to downstream queues (i.e., with higher index) will allow us to express all $G_{k i}(z)$ for a fixed $k$ in terms of functions $G_{k-1, j}(z)$, and finally in terms of the functions $G_{1 j}(z)$, which can be determined explicitly.

We begin by giving the equations for $G_{1 j}(z), j=1, \ldots, n$. Obviously

$$
G_{11}(z)=\mathbb{P}(M=1)=\pi_{1}
$$

indeed, after gate 1 has opened, $Q_{1}$ instantaneously has become empty. Now consider two successive gate openings in steady state, the latter one being an opening of gate $j$, and sum over all possible gates $i$ opened at the previous gate opening, to obtain 


$$
G_{1 j}(z)=\sum_{i=1}^{n} G_{1 i}(z) p_{i j} A_{i j 1}(z), \quad j \neq 1 .
$$

Here, we have used that $A_{i j 1}(z)$ is the generating function of the number of arrivals at $Q_{1}$ in the gate opening interval.

Notice that we can rewrite (3) as

$$
G_{1 j}(z)=\sum_{i=2}^{n} G_{1 i}(z) p_{i j} A_{i j 1}(z)+G_{11}(z) p_{1 j} A_{1 j 1}(z), \quad j \neq 1 .
$$

Introducing the $(n-1)$-dimensional vector

$$
\bar{G}_{1}(z):=\left(G_{12}(z), \ldots, G_{1 n}(z)\right),
$$

the $(n-1)$-dimensional vector

$$
R_{1}(z):=\left(p_{12} A_{121}(z), \ldots, p_{1 n} A_{1 n 1}(z)\right),
$$

and the matrix $P_{1}(z)$ with as $(i, j)$ element $p_{i j} A_{i j 1}(z)$, we can write (4) as

$$
\bar{G}_{1}(z)=\bar{G}_{1}(z) P_{1}(z)+G_{11}(z) R_{1}(z)
$$

and hence, with $I$ the matrix with ones on the diagonal and zeroes outside the diagonal, we have $\bar{G}_{1}(z)\left(I-P_{1}(z)\right)=G_{11}(z) R_{1}(z)$, yielding

$$
\bar{G}_{1}(z)=G_{11}(z) R_{1}(z)\left(I-P_{1}(z)\right)^{-1}
$$

All the terms on the right-hand side of (6) are known; in particular, $G_{11}(z)=\pi_{1}$ is given in (2). Hence we have determined $G_{11}(z), G_{12}(z), \ldots, G_{1 n}(z)$.

Now let us show how the terms $G_{k j}(z), j=1, \ldots, n$, are for $2 \leq k \leq n$ expressed in terms of $G_{k-1, i}(z), i=1, \ldots, n$. Considering two successive gate openings in steady state, the last one being of gate $j$, and summing over all possible gates $i$ for the first gate opening, we have for $k=2, \ldots, n, j \neq k$ :

$$
G_{k j}(z)=\sum_{i=1}^{n} G_{k i}(z) p_{i j} A_{i j k}(z)
$$

whereas

$$
G_{k k}(z)=\sum_{i=1}^{n} G_{k-1, i}(z) p_{i k} A_{i k, k-1}(z) .
$$

The explanation for the deviating terms $\left(G_{k-1, i}(z)\right.$ instead of $G_{k i}(z)$ and $A_{i k, k-1}(z)$ instead of $\left.A_{i k k}(z)\right)$ is that $Q_{k}$ has become empty right after an opening of gate $k$; so the total number present in $Q_{1}, \ldots, Q_{k}$ equals the total number present in $Q_{1}, \ldots, Q_{k-1}$ 
after the previous gate opening, plus the number of new arrivals in the first $k-1$ queues.

We can rewrite (7) as follows:

$$
G_{k j}(z)=\sum_{i \neq k} G_{k i}(z) p_{i j} A_{i j k}(z)+G_{k k}(z) p_{k j} A_{k j k}(z) .
$$

Introducing the $(n-1)$-dimensional vector

$$
\bar{G}_{k}(z):=\left(G_{k 1}(z), \ldots, G_{k, k-1}(z), G_{k, k+1}(z), \ldots, G_{k n}(z)\right),
$$

the $(n-1)$-dimensional vector

$$
R_{k}(z):=\left(p_{k 1} A_{k 1 k}(z), \ldots, p_{k, k-1} A_{k, k-1, k}(z), p_{k, k+1} A_{k, k+1, k}(z), \ldots, p_{k n} A_{k n k}(z)\right)
$$

and the matrix $P_{k}(z)$ with as $(i, j)$ element $p_{i j} A_{i j k}(z)$, we can write (9) as

$$
\bar{G}_{k}(z)=\bar{G}_{k}(z) P_{k}(z)+G_{k k}(z) R_{k}(z)
$$

yielding

$$
\bar{G}_{k}(z)=G_{k k}(z) R_{k}(z)\left(I-P_{k}(z)\right)^{-1}
$$

Introducing the column vector

$$
\begin{aligned}
C_{k-1}^{T}(z):= & \left(p_{1 k} A_{1 k, k-1}(z), \ldots, p_{k-2, k} A_{k-2, k, k-1}(z), p_{k k} A_{k k, k-1}(z), \ldots,\right. \\
& \left.p_{n k} A_{n k, k-1}(z)\right)^{T}
\end{aligned}
$$

we can rewrite (8) as

$$
G_{k k}(z)=\bar{G}_{k-1}(z) C_{k-1}^{T}(z)+G_{k-1, k-1}(z) p_{k-1, k} A_{k-1, k, k-1}(z) .
$$

We have thus expressed $\bar{G}_{k}(z)$ in terms of $G_{k k}(z)$ via (11), and $G_{k k}(z)$ in terms of $\bar{G}_{k-1}(z)$ and $G_{k-1, k-1}(z)$ via (12). Iterating, defining an empty product to be one and defining $\bar{G}_{0}(z) C_{0}^{T}(z)$ to equal $\pi_{1}$ for notational elegance, we obtain

$$
G_{k k}(z)=\sum_{i=0}^{k-1} \bar{G}_{i}(z) C_{i}^{T}(z) \prod_{j=i+1}^{k-1} p_{j, j+1} A_{j, j+1, j}(z) .
$$

By carefully studying the structure of the above recursions, and introducing

$$
H_{i}(z):=R_{i}(z)\left(I-P_{i}(z)\right)^{-1} C_{i}^{T}(z), \quad i=1, \ldots, n,
$$

the following is seen to hold:

$$
G_{k k}(z)=\pi_{1} \sum \prod_{i=1}^{k-1} F_{i}(z), \quad k=1, \ldots, n,
$$


where $\Sigma$ denotes a sum over the $2^{k-1}$ terms that arise when each $F_{i}(z), i=1, \ldots, k-1$, is either $H_{i}(z)$ or $p_{i, i+1} A_{i, i+1, i}(z)$. For example, for $k=3$ we get

$$
\begin{aligned}
G_{33}(z)= & \pi_{1}\left[H_{1}(z) H_{2}(z)+H_{1}(z) p_{23} A_{232}(z)+p_{12} A_{121}(z) H_{2}(z)\right. \\
& \left.+p_{12} A_{121}(z) p_{23} A_{232}(z)\right] .
\end{aligned}
$$

With this explicit expression (14) for the $G_{k k}(z)$, and expression (11) for $\bar{G}_{k}(z)$, we have a recipe to determine all $G_{k j}(z)$ explicitly, for $k, j=1, \ldots, n$.

Example Let us consider the special case in which $p_{i j} \equiv q_{j}, \forall i, j$, and $A_{i j k}(z)=$ : $\hat{A}_{j k}(z), \forall i, j, k$. Viz., the Markov renewal process that determines the gate openings and the intervals in between has a simple structure: Each time the next gate opening is of gate $j$ with probability $q_{j}$, and the interval length until the next opening also only depends on $j$. In this case, we can obtain a simple expression for $\mathbb{E}\left[z^{X_{(k)}}\right]=$ $\sum_{j=1}^{n} G_{k j}(z)$. We have

$$
G_{11}(z)=\pi_{1}=q_{1}
$$

and from (3)

$$
G_{1 j}(z)=q_{j} \hat{A}_{j 1}(z) \sum_{i=1}^{n} G_{1 i}(z), \quad j=2, \ldots, n
$$

Hence

$$
\mathbb{E}\left[z^{X_{(1)}}\right]=\sum_{j=1}^{n} G_{1 j}(z)=q_{1}+\sum_{j=2}^{n} q_{j} \hat{A}_{j 1}(z) \mathbb{E}\left[z^{X_{(1)}}\right]
$$

yielding

$$
\mathbb{E}\left[z^{X_{(1)}}\right]=\frac{q_{1}}{1-\sum_{j=2}^{n} q_{j} \hat{A}_{j 1}(z)} .
$$

Furthermore, from (7) and (8),

$$
\begin{aligned}
& G_{k j}(z)=q_{j} \hat{A}_{j k}(z) \sum_{i=1}^{n} G_{k i}(z) \\
& G_{k k}(z)=q_{k} \hat{A}_{k, k-1}(z) \sum_{i=1}^{n} G_{k-1, i}(z),
\end{aligned}
$$

leading to the following recursive expression for $\mathbb{E}\left[z^{X_{(k)}}\right]$ in terms of $\mathbb{E}\left[z^{X_{(k-1)}}\right]$ :

$$
\mathbb{E}\left[z^{X_{(k)}}\right]=\frac{q_{k} \hat{A}_{k, k-1}(z)}{1-\sum_{j \neq k} q_{j} \hat{A}_{j k}(z)} \mathbb{E}\left[z^{X_{(k-1)}}\right]
$$


Via iteration we obtain

$$
\mathbb{E}\left[z^{X_{(k)}}\right]=\prod_{i=1}^{k} \frac{q_{i} \hat{A}_{i, i-1}(z)}{1-\sum_{j \neq i} q_{j} \hat{A}_{j k}(z)},
$$

where $\hat{A}_{10}(z):=1$.

Notice that (22) represents a decomposition property: The generating function is a product of $k$ terms, all of which are generating functions of random variables, and this implies that $X_{(k)}$ can be represented as the sum of $k$ independent random variables, cf. $[6,7]$. In the special case that arrivals only occur at $Q_{1}$, and that the generating function of the number of arrivals in all gate intervals is the same, to be denoted by $\hat{A}(z)$, we have

$$
\mathbb{E}\left[z^{X_{(k)}}\right]=\hat{A}^{k-1}(z) \prod_{i=1}^{k} \frac{q_{i}}{1-\hat{A}(z)\left(1-q_{i}\right)} .
$$

When we consider for this case the steady-state number of customers $N_{(n)}$ just before a gate opening, we get a slightly more elegant expression. Observing that $\mathbb{E}\left[z^{N_{(n)}}\right]=$ $\mathbb{E}\left[z^{X_{(n)}}\right] \hat{A}(z)$, we can write

$$
\mathbb{E}\left[z^{N_{(n)}}\right]=\prod_{i=1}^{n} \frac{q_{i} \hat{A}(z)}{1-\hat{A}(z)\left(1-q_{i}\right)} .
$$

This shows that $N_{(n)}$ is distributed like the sum of $n$ independent geometric sums of numbers of arrivals during one gate interval. In particular,

$$
\begin{aligned}
\mathbb{E} N_{(n)} & =\mathbb{E} A \sum_{i=1}^{n} \frac{1}{q_{i}} \\
\operatorname{Var} N_{(n)} & =(\mathbb{E} A)^{2} \sum_{i=1}^{n}\left(\frac{1}{q_{i}^{2}}-\frac{1}{q_{i}}\right)+\operatorname{Var} A \sum_{i=1}^{n} \frac{1}{q_{i}} .
\end{aligned}
$$

$A$ denoting the number of arrivals during one gate interval.

The special choice $\hat{A}(z)=z$ (one arrival in each gate interval) yields

$$
\mathbb{E}\left[z^{X_{(k)}}\right]=z^{k-1} \prod_{i=1}^{k} \frac{q_{i}}{1-\left(1-q_{i}\right) z},
$$

and hence $X_{(k)}=k-1+\sum_{i=1}^{k} B_{i}$, where $B_{i} \sim \operatorname{geom}\left(1-q_{i}\right)$ for $i=1, \ldots, k$, and $\mathbb{E} X_{(k)}=k-1+\sum_{i=1}^{k} \frac{1-q_{i}}{q_{i}}=\sum_{i=1}^{k} \frac{1}{q_{i}}-1$.

The special choice $\hat{A}(z)=\frac{\mu}{\mu+\lambda(1-z)}$ (a Poisson distributed number of arrivals in an $\exp (\mu)$ distributed interval, giving rise to a geometrically distributed number of arrivals in a gate interval) yields 


$$
\mathbb{E}\left[z^{X_{(k)}}\right]=\left(\frac{\mu}{\mu+\lambda(1-z)}\right)^{k-1} \prod_{i=1}^{k} \frac{q_{i}(\mu+\lambda(1-z))}{q_{i} \mu+\lambda(1-z)},
$$

and hence $X_{(k)}=F_{k-1}+\sum_{i=1}^{k} C_{i}$, where $F_{k-1}$ is negative binomially distributed with parameters $k-1$ and $\frac{\lambda}{\mu+\lambda}$ and where $C_{i}$ equals zero with probability $q_{i}$ and is $\operatorname{geom}\left(\frac{q_{i} \mu}{q_{i} \mu+\lambda}\right)$ distributed with probability $1-q_{i}, i=1, \ldots, k$. Hence $\mathbb{E} X_{(k)}=$ $(k-1) \frac{\lambda}{\mu}+\sum_{i=1}^{k}\left(1-q_{i}\right) \frac{\lambda}{q_{i} \mu}$. More generally, it follows from (23) that $\mathbb{E} X_{(k)}=$ $\left[k-1+\sum_{i=1}^{k} \frac{1-q_{i}}{q_{i}}\right] \mathbb{E} A$.

\section{Optimization under constraints}

In this section we consider three optimization problems, which are very similar to optimization problems studied in [6] for the special case of exponential gate openings. Our goal is to design an efficient ASIP system. We restrict ourselves to the case, leading to (24), in which arrivals only occur in $Q_{1}$ while the generating function of the number of arrivals in all gate intervals is the same. For this case, we pose the question which choice of $\left(q_{1}, \ldots, q_{n}\right)$, with $\sum_{i=1}^{n} q_{i}=1$, (i) minimizes the mean number of customers $N_{(n)}$ just before a gate opening, (ii) minimizes the variance of $N_{(n)}$, and (iii) maximizes the probability of zero load (an empty system).

Optimization problem (i): minimization of the mean number of customers

It follows from (25) that the minimization of the mean number of customers amounts to minimizing $\sum_{i=1}^{n} \frac{1}{q_{i}}$, subject to $\sum_{i=1}^{n} q_{i}=1$. This optimization problem is a special case of the class of resource allocation problems with a separable convex objective function i.e., the objective function can be separated into $n$ terms, the $i$ th one being a function of $q_{i}$ only, that is convex in $q_{i}, i=1, \ldots, n$. We wish to minimize this separable convex function under a linear constraint. This class of problems is extensively studied in [4]. In particular, if $f$ is convex then

$$
f(1 / n)=f\left(\frac{1}{n} \sum_{i=1}^{n} q_{i}\right) \leq \frac{1}{n} \sum_{i=1}^{n} f\left(q_{i}\right)
$$

and so $\sum_{i=1}^{n} f(1 / n) \leq \sum_{i=1}^{n} f\left(q_{i}\right)$ for any $q_{i} \geq 0$ such that $\sum_{i=1}^{n} q_{i}=1$; thus the optimal solution of our minimization problem is $q_{1}=\cdots=q_{n}=\frac{1}{n}$.

It should be noted that the mean number of customers just before a gate opening is readily expressed in the steady-state mean number of customers at an arbitrary epoch; just subtract the mean number of arrivals in a residual gate opening interval. The latter is linearly related to the mean time in system via Little's law. Hence the above optimization problem also sheds light on the minimization of time in system.

Optimization problem (ii): minimization of the variance of the number of customers It follows from (26) that the minimization of the variance of the number of customers amounts to minimizing $\sum_{i=1}^{n}\left[\left(\frac{1}{q_{i}^{2}}-\frac{1}{q_{i}}\right)(\mathbb{E} A)^{2}+\frac{1}{q_{i}} \operatorname{Var} A\right]$. The same reasoning as for (i) applies; we again are faced with a separable convex objective function, and again the optimal solution is $q_{1}=\cdots=q_{n}=\frac{1}{n}$. 
Optimization problem (iii): maximization of the probability of an empty system It follows from (24) that the maximization of the probability of an empty system amounts to maximizing $\prod_{i=1}^{n} \frac{q_{i} \hat{A}(0)}{1-\left(1-q_{i}\right) \hat{A}(0)}$, and hence to minimizing $\sum_{i=1}^{n} \ln \left[\frac{1-\hat{A}(0)}{q_{i}}+\right.$ $\hat{A}(0)]$. The same reasoning as for (i) and (ii) applies once again; we have a separable convex objective function, and again the optimal solution is $q_{1}=\cdots=q_{n}=\frac{1}{n}$.

\section{Some two-queue results}

In this section we study the two-queue case in some more detail. In that case one can sometimes determine the joint queue length distribution at gate opening intervals. In Sect. 5.1 we determine the joint queue length distribution at gate openings for a specific choice of the $p_{i j}$ and the same arrival distributions for gate 1 intervals and gate 2 intervals. In Sect. 5.2 we determine the joint queue length distribution for the case in which, when the gate of $Q_{1}$ opens, only a binomially distributed number of the customers in $Q_{1}$ moves to $Q_{2}$. These two-queue studies not only lead to more detailed results, they also sometimes give an indication of the limitations of our approach. For example, if one would not only at $Q_{1}$, but also at $Q_{2}$, allow a binomially distributed number of customers to leave when its gate opens, then a functional equation in the two-dimensional queue length probability generating function results, which seems very difficult to analyze exactly.

\subsection{Joint queue length distribution}

Let us consider the problem of determining the generating function of the steady-state joint queue length distribution right after gate openings, $G\left(z_{1}, z_{2}\right)=\mathbb{E}\left[z_{1}^{X_{1}} z_{2}^{X_{2}}\right]$. Take $n=2$; take only arrivals at $Q_{1}$, with generating function $A(z)$ of the number of arrivals per gate opening, regardless whether it is an opening of gate 1 or of gate 2 ; and take fixed gate opening probabilities $p_{i j} \equiv q_{j}$. Realizing that, with $X_{i}^{(r)}$ the number of customers in $Q_{i}$ right after the $r$ th gate opening, and with $A_{r+1}$ the number of arrivals in the interval between the $r$ th and $(r+1)$ st gate openings,

$$
X_{1}^{(r+1)}=0, \quad X_{2}^{(r+1)}=X_{1}^{(r)}+A_{r+1}+X_{2}^{(r)},
$$

if the $(r+1)$ st gate opening is of gate 1 , and

$$
X_{1}^{(r+1)}=X_{1}^{(r)}+A_{r+1}, \quad X_{2}^{(r+1)}=0,
$$

if the $(r+1)$ st gate opening is of gate 2 , we obtain in steady state:

$$
G\left(z_{1}, z_{2}\right)=q_{1} A\left(z_{2}\right) G\left(z_{2}, z_{2}\right)+q_{2} A\left(z_{1}\right) G\left(z_{1}, 1\right) .
$$

Actually we already know $G\left(z_{1}, 1\right)$, which equals $\mathbb{E}\left[z_{1}^{X_{(1)}}\right]$; but it also follows from (29) by putting $z_{2}=1$. We also already know $G\left(z_{1}, z_{1}\right)$, which equals $\mathbb{E}\left[z_{1}^{X_{(2)}}\right]$; but it 
also follows from (29) by putting $z_{2}=z_{1}$. We find

$$
\begin{aligned}
G\left(z_{1}, 1\right) & =\frac{q_{1}}{1-q_{2} A\left(z_{1}\right)}, \\
G\left(z_{1}, z_{1}\right) & =\frac{q_{1} q_{2} A\left(z_{1}\right)}{1-q_{2} A\left(z_{1}\right)} \frac{1}{1-q_{1} A\left(z_{1}\right)}, \\
G\left(z_{1}, z_{2}\right) & =\frac{q_{1} q_{2} A\left(z_{2}\right)}{1-q_{2} A\left(z_{2}\right)} \frac{q_{1} A\left(z_{2}\right)}{1-q_{1} A\left(z_{2}\right)}+\frac{q_{1} q_{2} A\left(z_{1}\right)}{1-q_{2} A\left(z_{1}\right)} .
\end{aligned}
$$

Remark One could extend the above analysis to the case of arrivals at both queues, and different PGFs for different gate openings. However, this comes at the expense of messier expressions, and we have decided not to include this case in the paper.

One could also in principle analyze the steady-state queue length PGF at an arbitrary epoch. One would then have to average over different gate opening intervals. However, the arrival process must then first be specified in more detail; do arrivals all take place at the beginning of a gate opening interval, or at the end, or maybe according to a Poisson process?

\subsection{Binomial movements}

Consider the case of $n=2$ queues in series, with the special feature that, when the gate of $Q_{1}$ opens, each customer present in $Q_{1}$ (independently from the other customers) moves with probability $a_{1}>0$ to $Q_{2}$, and stays with probability $1-a_{1}$ in $Q_{1}$. We restrict ourselves to the case of a Poisson arrival process, with rate $\lambda$, at $Q_{1}$, and no external arrivals at $Q_{2}$; moreover, we assume that gate openings at $Q_{i}$ occur after i.i.d., exponentially distributed intervals with mean $1 / \mu_{i}, i=1,2$. Denoting by $X_{i}(t)$ the number of customers in $Q_{i}$ at time $t, i=1,2$, and by $X_{1}^{\text {bin }}(t)$ the number of customers who do move from $Q_{1}$ to $Q_{2}$ at a gate opening of $Q_{1}$ that takes place at time $t$, we can write (suppressing initial conditions; we shall anyway soon turn to the steady-state situation)

$$
\begin{aligned}
\mathbb{E}\left[z_{1}^{X_{1}(t+h)} z_{2}^{X_{2}(t+h)}\right]= & \left(1-\left(\lambda+\mu_{1}+\mu_{2}\right) h\right) \mathbb{E}\left[z_{1}^{X_{1}(t)} z_{2}^{X_{2}(t)}\right] \\
& +\lambda h z_{1} \mathbb{E}\left[z_{1}^{X_{1}(t)} z_{2}^{X_{2}(t)}\right] \\
& +\mu_{1} h \mathbb{E}\left[z_{1}^{X_{1}(t)-X_{1}^{\text {bin }}(t)} z_{2}^{X_{2}(t)+X_{1}^{\text {bin }}(t)}\right] \\
& +\mu_{2} h \mathbb{E}\left[z_{1}^{X_{1}(t)}\right]+o(h), \quad h \downarrow 0,
\end{aligned}
$$

leading to

$$
\begin{aligned}
\frac{\mathrm{d}}{\mathrm{d} t} \mathbb{E}\left[z_{1}^{X_{1}(t)} z_{2}^{X_{2}(t)}\right]= & -\left(\lambda+\mu_{1}+\mu_{2}\right) \mathbb{E}\left[z_{1}^{X_{1}(t)} z_{2}^{X_{2}(t)}\right] \\
& +\lambda z_{1} \mathbb{E}\left[z_{1}^{X_{1}(t)} z_{2}^{X_{2}(t)}\right]
\end{aligned}
$$




$$
\begin{aligned}
& +\mu_{1} \mathbb{E}\left[\left(\left(1-a_{1}\right) z_{1}+a_{1} z_{2}\right)^{X_{1}(t)} z_{2}^{X_{2}(t)}\right] \\
& +\mu_{2} \mathbb{E}\left[z_{1}^{X_{1}(t)}\right] .
\end{aligned}
$$

Denoting the probability generating function of the joint distribution of the steady-state queue length vector $\left(X_{1}, X_{2}\right)$ by $H\left(z_{1}, z_{2}\right)$, we have

$$
\left[\mu_{1}+\mu_{2}+\lambda\left(1-z_{1}\right)\right] H\left(z_{1}, z_{2}\right)=\mu_{1} H\left(\left(1-a_{1}\right) z_{1}+a_{1} z_{2}, z_{2}\right)+\mu_{2} H\left(z_{1}, 1\right)
$$

We shall first obtain $H\left(z_{1}, 1\right)$. Substituting $z_{2}=1$ into (35) yields

$$
\left[\mu_{1}+\mu_{2}+\lambda\left(1-z_{1}\right)\right] H\left(z_{1}, 1\right)=\mu_{1} H\left(\left(1-a_{1}\right) z_{1}+a_{1}, 1\right)+\mu_{2} H\left(z_{1}, 1\right),
$$

and hence

$$
H\left(z_{1}, 1\right)=\frac{\mu_{1}}{\mu_{1}+\lambda\left(1-z_{1}\right)} H\left(\left(1-a_{1}\right) z_{1}+a_{1}, 1\right) .
$$

Iteration of this relation gives

$$
H\left(z_{1}, 1\right)=\prod_{j=0}^{\infty} \frac{\mu_{1}}{\mu_{1}+\lambda\left(1-z_{1}\right)\left(1-a_{1}\right)^{j}}
$$

This infinite product is said to converge iff $\sum_{j=0}^{\infty}\left(1-\frac{\mu_{1}}{\mu_{1}+\lambda\left(1-z_{1}\right)\left(1-a_{1}\right)^{j}}\right)<\infty$, and hence the infinite product indeed converges if $0<a_{1}<1$. If $a_{1}=1$ one obtains $H\left(z_{1}, 1\right)=\frac{\mu_{1}}{\mu_{1}+\lambda\left(1-z_{1}\right)}$. This is not a surprising result; it is the generating function of the number of Poisson $(\lambda)$ arrivals during an $\exp (\mu)$ interval. According to PASTA, it also equals the generating function of the steady-state queue length distribution of $Q_{1}$. Observing that $\frac{\mu_{1}}{\mu_{1}+\lambda\left(1-z_{1}\right)\left(1-a_{1}\right)^{j}}$ is the probability generating function of a geometrically distributed random variable with success parameter $\frac{\lambda\left(1-a_{1}\right)^{j}}{\mu_{1}+\lambda\left(1-a_{1}\right)^{j}}$, one can write

$$
X_{1} \stackrel{d}{=} \sum_{j=0}^{\infty} H_{j}
$$

where all $H_{j}$ are independent, $H_{j}$ being geometrically distributed with success parameter $\frac{\lambda\left(1-a_{1}\right)^{j}}{\mu_{1}+\lambda\left(1-a_{1}\right)^{j}}$.

Having determined $H\left(z_{1}, 1\right)$, we now turn to the determination of $H\left(z_{1}, z_{2}\right)$. It follows from (35) that

$$
H\left(z_{1}, z_{2}\right)=Y_{1}\left(z_{1}\right) H\left(\left(1-a_{1}\right) z_{1}+a_{1} z_{2}, z_{2}\right)+Y_{0}\left(z_{1}\right)
$$

where

$$
Y_{1}\left(z_{1}\right):=\frac{\mu_{1}}{\mu_{1}+\mu_{2}+\lambda\left(1-z_{1}\right)}, \quad Y_{0}\left(z_{1}\right):=\frac{\mu_{2}}{\mu_{1}+\mu_{2}+\lambda\left(1-z_{1}\right)} H\left(z_{1}, 1\right) .
$$


Iteration of (40) gives

$$
H\left(z_{1}, z_{2}\right)=\sum_{j=0}^{\infty} Y_{0}\left(f_{j}\left(z_{1}, z_{2}\right)\right) \prod_{i=0}^{j-1} Y_{1}\left(f_{i}\left(z_{1}, z_{2}\right)\right)
$$

an empty product being equal to one and $f_{i}\left(z_{1}, z_{2}\right):=\left(1-a_{1}\right)^{i} z_{1}+[1-(1-$ $\left.\left.a_{1}\right)^{i}\right] z_{2}, i=0,1, \ldots$ Using d'Alembert's ratio test one can show that this infinite sum converges. In fact, the sum converges geometrically fast. Indeed, since $a_{1}>0$, one has $f_{j}\left(z_{1}, z_{2}\right) \rightarrow z_{2}$, and the ratio of two successive terms in the sum $H\left(z_{1}, z_{2}\right)$, which is given by $\frac{Y_{0}\left(f_{j+1}\left(z_{1}, z_{2}\right)\right)}{Y_{0}\left(f_{j}\left(z_{1}, z_{2}\right)\right)} Y_{1}\left(f_{j}\left(z_{1}, z_{2}\right)\right)$, is for large $j$ bounded by $\mu_{1} /\left(\mu_{1}+\mu_{2}\right)$.

Above, we have restricted ourselves to the case of a Poisson arrival process, with rate $\lambda$, at $Q_{1}$, and no external arrivals at $Q_{2}$; moreover, we assumed that gate openings at $Q_{i}$ occur after i.i.d. exponentially distributed intervals with mean $1 / \mu_{i}, i=1,2$. Let us now turn to the more general case of Sect. 2, in which gate openings are determined by a Markov renewal process, and where a gate opening of $Q_{i}$ is with probability $p_{i j}$ followed by a gate opening of $Q_{j}$, while $A_{i j}\left(z_{1}, z_{2}\right)$ is the generating function of the numbers of arrivals in $Q_{1}$ and $Q_{2}$ during the period in between those two successive gate openings. Considering the steady-state joint distribution of the numbers of customers $\left(X_{1}, X_{2}\right)$ immediately after gate openings, and letting (cf. (2))

$$
G_{i}\left(z_{1}, z_{2}\right):=\mathbb{E}\left[z_{1}^{X_{1}} z_{2}^{X_{2}} I(M=i)\right], \quad i=1,2,
$$

it is easily seen by observing the system at two successive gate openings that

$$
\begin{aligned}
G_{1}\left(z_{1}, z_{2}\right)= & p_{11} A_{11}\left(\left(1-a_{1}\right) z_{1}+a_{1} z_{2}, z_{2}\right) G_{1}\left(\left(1-a_{1}\right) z_{1}+a_{1} z_{2}, z_{2}\right) \\
& +p_{21} A_{21}\left(\left(1-a_{1}\right) z_{1}+a_{1} z_{2}, z_{2}\right) G_{2}\left(\left(1-a_{1}\right) z_{1}+a_{1} z_{2}, z_{2}\right), \\
G_{2}\left(z_{1}, z_{2}\right)= & p_{12} A_{12}\left(z_{1}, 1\right) G_{1}\left(z_{1}, 1\right)+p_{22} A_{22}\left(z_{1}, 1\right) G_{2}\left(z_{1}, 1\right)
\end{aligned}
$$

It is immediately obvious from (44) that $G_{2}\left(z_{1}, z_{2}\right)$ does not depend on $z_{2}$, as we could have expected, because $Q_{2}$ becomes empty after a gate opening at $Q_{2}$. Hence, it follows from (44) that

$$
G_{2}\left(z_{1}, z_{2}\right)=G_{2}\left(z_{1}, 1\right)=\frac{p_{12} A_{12}\left(z_{1}, 1\right)}{1-p_{22} A_{22}\left(z_{1}, 1\right)} G_{1}\left(z_{1}, 1\right)
$$

Plugging $z_{2}=1$ in (43) and using (45) gives

$$
\begin{aligned}
G_{1}\left(z_{1}, 1\right)= & p_{11} A_{11}\left(\left(1-a_{1}\right) z_{1}+a_{1}, 1\right) G_{1}\left(\left(1-a_{1}\right) z_{1}+a_{1}, 1\right) \\
& +p_{21} A_{21}\left(\left(1-a_{1}\right) z_{1}+a_{1}, 1\right) \frac{p_{12} A_{12}\left(\left(1-a_{1}\right) z_{1}+a_{1}, 1\right)}{1-p_{22} A_{22}\left(\left(1-a_{1}\right) z_{1}+a_{1}, 1\right)} \\
& G_{1}\left(\left(1-a_{1}\right) z_{1}+a_{1}, 1\right),
\end{aligned}
$$


which can be written as

$$
G_{1}\left(z_{1}, 1\right)=L\left(z_{1}\right) G_{1}\left(\left(1-a_{1}\right) z_{1}+a_{1}, 1\right)
$$

with an obvious choice of the function $L(\cdot)$. Iteration readily yields that

$$
G_{1}\left(z_{1}, 1\right)=\prod_{j=0}^{\infty} L\left(\mathrm{~d}^{(j)}\left(z_{1}\right)\right)
$$

where

$$
\mathrm{d}^{(j)}\left(z_{1}\right):=\left(1-a_{1}\right)^{j} z_{1}+1-\left(1-a_{1}\right)^{j}, \quad j=0,1, \ldots
$$

The infinite product converges iff the corresponding infinite sum $\sum_{j=0}^{\infty}[1-$ $\left.L\left(\mathrm{~d}^{(j)}\left(z_{1}\right)\right)\right]$ converges. The latter sum converges geometrically fast. This can be seen by making the following two observations. Observation (i): $L\left(z_{1}\right)$ has the meaning of a probability generating function. Indeed, distinguish between the possibility that a gate opening of $Q_{1}$ is followed by another gate opening of $Q_{1}$ (probability $p_{11}$ ) and the possibility that it is followed by a gate opening of $Q_{2}$, followed by a geometric $\left(p_{22}\right)$ number of gate openings of $Q_{2}$, and finally again a gate opening of $Q_{1}$. Observation (ii): $1-\mathrm{d}^{(j)}\left(z_{1}\right)=\left(1-a_{1}\right)^{j}\left(1-z_{1}\right)$ converges geometrically fast to 0 .

Having determined $G_{1}\left(z_{1}, 1\right)$ and hence, using (45), $G_{2}\left(z_{1}, z_{2}\right)=G_{2}\left(z_{1}, 1\right)$, we substitute the result in (43), obtaining

$$
G_{1}\left(z_{1}, z_{2}\right)=K_{1}\left(z_{1}, z_{2}\right) G_{1}\left(\left(1-a_{1}\right) z_{1}+a_{1} z_{2}, z_{2}\right)+K_{0}\left(z_{1}, z_{2}\right),
$$

where

$$
\begin{aligned}
K_{1}\left(z_{1}, z_{2}\right):= & p_{11} A_{11}\left(\left(1-a_{1}\right) z_{1}+a_{1} z_{2}, z_{2}\right), \\
K_{0}\left(z_{1}, z_{2}\right):= & p_{21} A_{21}\left(\left(1-a_{1}\right) z_{1}+a_{1} z_{2}, z_{2}\right) \frac{p_{12} A_{12}\left(\left(1-a_{1}\right) z_{1}+a_{1} z_{2}, 1\right)}{1-p_{22} A_{22}\left(\left(1-a_{1}\right) z_{1}+a_{1} z_{2}, 1\right)} \\
& G_{1}\left(\left(1-a_{1}\right) z_{1}+a_{1} z_{2}, 1\right) .
\end{aligned}
$$

Iteration of (50) gives

$$
G_{1}\left(z_{1}, z_{2}\right)=\sum_{j=0}^{\infty} K_{0}\left(f_{j}\left(z_{1}, z_{2}\right), z_{2}\right) \prod_{i=0}^{j-1} K_{1}\left(f_{i}\left(z_{1}, z_{2}\right), z_{2}\right) .
$$

Again d'Alembert's ratio test readily shows the convergence of the infinite sum, by using that $\left|K_{1}\left(z_{1}, z_{2}\right)\right|<p_{11}$. Finally, notice that $G_{1}\left(z_{2}, z_{2}\right)$, which is the generating function of the total number of customers $X_{(2)}=X_{1}+X_{2}$ in the two queues just after gate openings of $Q_{1}$, follows by substituting $z_{1}=z_{2}$ in (53). Since $f_{j}\left(z_{2}, z_{2}\right) \equiv z_{2}$, that formula degenerates into 


$$
G_{1}\left(z_{2}, z_{2}\right)=\frac{K_{0}\left(z_{2}, z_{2}\right)}{1-K_{1}\left(z_{2}, z_{2}\right)} .
$$

After some calculations, this expression is seen to agree with the expression for $G_{21}\left(z_{2}\right)$ that can be derived from (7). This agreement may at first sight seem strange, as we have binomial movements in the present subsection. However, notice that we compare $G_{1}\left(z_{2}, z_{2}\right)$ and $G_{21}\left(z_{2}\right)$, both giving the total number of customers in both queues. It then does not matter whether some of them are still in $Q_{1}$ after a gate opening of $Q_{1}$.

\subsection{An ASIP model with a renewal arrival process at $Q_{1}$}

In this subsection we consider the case in which arrivals only take place at $Q_{1}$, and follow a renewal process: successive interarrival times are i.i.d., with distribution $A(\cdot)$ and Laplace-Stieltjes transform $\alpha(\cdot)$. We restrict ourselves to $n=2$ queues. We furthermore restrict ourselves to the case in which openings of the gate of $Q_{i}$ occur at i.i.d. $\exp \left(\mu_{i}\right)$ distributed intervals, independent of each other and independent of the arrival intervals.

Let $\left(Y_{n, 1}, Y_{n, 2}\right)$ denote the vector of numbers of customers in $\left(Q_{1}, Q_{2}\right)$ just before the $n$th arrival at $Q_{1}, n=1,2, \ldots$ Let $A_{n}$ denote the arrival interval between customers $n-1$ and $n$. We need to distinguish between the following five cases:

(i) No gate opening in $A_{n}$. This event has probability $\alpha\left(\mu_{1}+\mu_{2}\right)$; and $\left(Y_{n, 1}, Y_{n, 2}\right)=$ $\left(Y_{n-1,1}+1, Y_{n-1,2}\right)$.

(ii) No openings of gate 1 and at least one opening of gate 2 in $A_{n}$. This event has probability $\alpha\left(\mu_{1}\right)-\alpha\left(\mu_{1}+\mu_{2}\right)$; and $\left(Y_{n, 1}, Y_{n, 2}\right)=\left(Y_{n-1,1}+1,0\right)$.

(iii) No openings of gate 2 and at least one opening of gate 1 in $A_{n}$. This event has probability $\alpha\left(\mu_{2}\right)-\alpha\left(\mu_{1}+\mu_{2}\right)$; and $\left(Y_{n, 1}, Y_{n, 2}\right)=\left(0, Y_{n-1,1}+1+Y_{n-1,2}\right)$.

(iv) Both gates open at least once in $A_{n}$; the first opening of gate 1 occurs after the last opening of gate 2 . This event has probability $\alpha\left(\mu_{1}+\mu_{2}\right)-\frac{\mu_{2}}{\mu_{2}-\mu_{1}} \alpha\left(\mu_{2}\right)-$ $\frac{\mu_{1}}{\mu_{1}-\mu_{2}} \alpha\left(\mu_{1}\right) ;$ and $\left(Y_{n, 1}, Y_{n, 2}\right)=\left(0, Y_{n-1,1}+1\right)$.

(v) Both gates open at least once in $A_{n}$; but the first opening of gate 1 occurs before the last opening of gate 2 . This event has probability $1-\frac{\mu_{2}}{\mu_{2}-\mu_{1}} \alpha\left(\mu_{1}\right)-\frac{\mu_{1}}{\mu_{1}-\mu_{2}} \alpha\left(\mu_{2}\right)$, as can, for example, be seen by writing the probability of this event as the probability that the sum of an $\exp \left(\mu_{1}\right)$ plus an $\exp \left(\mu_{2}\right)$ random variable is less than $A_{n}$. We now have $\left(Y_{n, 1}, Y_{n, 2}\right)=(0,0)$; notice that this is the only way to get into the state $(0,0)$. Restricting ourselves to steady-state queue lengths just before arrivals, to be denoted by $\left(Y_{1}, Y_{2}\right)$, and introducing their generating function $L\left(z_{1}, z_{2}\right):=\mathbb{E}\left[z_{1}^{Y_{1}} z_{2}^{Y_{2}}\right]$, we obtain

$$
\begin{aligned}
L\left(z_{1}, z_{2}\right)= & \alpha\left(\mu_{1}+\mu_{2}\right) z_{1} L\left(z_{1}, z_{2}\right) \\
& +\left(\alpha\left(\mu_{1}\right)-\alpha\left(\mu_{1}+\mu_{2}\right)\right) z_{1} L\left(z_{1}, 1\right) \\
& +\left(\alpha\left(\mu_{2}\right)-\alpha\left(\mu_{1}+\mu_{2}\right)\right) z_{2} L\left(z_{2}, z_{2}\right) \\
& +\left[\alpha\left(\mu_{1}+\mu_{2}\right)-\frac{\mu_{2}}{\mu_{2}-\mu_{1}} \alpha\left(\mu_{2}\right)-\frac{\mu_{1}}{\mu_{1}-\mu_{2}} \alpha\left(\mu_{1}\right)\right] z_{2} L\left(z_{2}, 1\right) \\
& +1-\frac{\mu_{2}}{\mu_{2}-\mu_{1}} \alpha\left(\mu_{1}\right)-\frac{\mu_{1}}{\mu_{1}-\mu_{2}} \alpha\left(\mu_{2}\right) .
\end{aligned}
$$


Taking all $L\left(z_{1}, z_{2}\right)$ terms together, and introducing

$$
\zeta:=1-\frac{\mu_{2}}{\mu_{2}-\mu_{1}} \alpha\left(\mu_{1}\right)-\frac{\mu_{1}}{\mu_{1}-\mu_{2}} \alpha\left(\mu_{2}\right),
$$

(which actually is $L(0,0)=P\left(Y_{1}=0, Y_{2}=0\right)$; see above) and

$$
\begin{gathered}
\omega:=\alpha\left(\mu_{1}+\mu_{2}\right)-\frac{\mu_{2}}{\mu_{2}-\mu_{1}} \alpha\left(\mu_{2}\right)-\frac{\mu_{1}}{\mu_{1}-\mu_{2}} \alpha\left(\mu_{1}\right) \\
=\alpha\left(\mu_{1}+\mu_{2}\right)-\alpha\left(\mu_{1}\right)-\alpha\left(\mu_{2}\right)+1-\zeta,
\end{gathered}
$$

we obtain

$$
\begin{aligned}
\left(1-\alpha\left(\mu_{1}+\mu_{2}\right) z_{1}\right) L\left(z_{1}, z_{2}\right)= & \left(\alpha\left(\mu_{1}\right)-\alpha\left(\mu_{1}+\mu_{2}\right)\right) z_{1} L\left(z_{1}, 1\right) \\
& +\left(\alpha\left(\mu_{2}\right)-\alpha\left(\mu_{1}+\mu_{2}\right)\right) z_{2} L\left(z_{2}, z_{2}\right) \\
& +\omega z_{2} L\left(z_{2}, 1\right)+\zeta
\end{aligned}
$$

Substitution of $z_{2}=1$ in (56), and using the fact that $\alpha\left(\mu_{2}\right)-\alpha\left(\mu_{1}+\mu_{2}\right)+\zeta+\omega=$ $1-\alpha\left(\mu_{1}\right)$ yields

$$
\left(1-\alpha\left(\mu_{1}+\mu_{2}\right) z_{1}\right) L\left(z_{1}, 1\right)=\left(\alpha\left(\mu_{1}\right)-\alpha\left(\mu_{1}+\mu_{2}\right)\right) z_{1} L\left(z_{1}, 1\right)+1-\alpha\left(\mu_{1}\right),
$$

and hence

$$
L\left(z_{1}, 1\right)=E\left[z_{1}^{Y_{1}}\right]=\frac{1-\alpha\left(\mu_{1}\right)}{1-\alpha\left(\mu_{1}\right) z_{1}} .
$$

The marginal distribution of $Y_{1}$ is hence geometric. The explanation is that $Y_{1}$ increases by 1 for a geometrically distributed number of arrival intervals (with parameter $\alpha\left(\mu_{1}\right)$, which is the probability that gate 1 does not close during an arrival interval), and then falls back to zero.

Substituting $z_{1}=z_{2}$ in (56) allows us to express $L\left(z_{2}, z_{2}\right)$ in terms of $L\left(z_{2}, 1\right)$ :

$$
\left(1-\alpha\left(\mu_{2}\right) z_{2}\right) L\left(z_{2}, z_{2}\right)=\left(\alpha\left(\mu_{1}\right)-\alpha\left(\mu_{1}+\mu_{2}\right)\right) z_{2} L\left(z_{2}, 1\right)+\zeta+\omega z_{2} L\left(z_{2}, 1\right)
$$

yielding the following expression for the generating function of the total number of customers in the system just before an arrival at $Q_{1}$ :

$$
L\left(z_{2}, z_{2}\right)=\frac{\left(\alpha\left(\mu_{1}\right)-\alpha\left(\mu_{1}+\mu_{2}\right)+\omega\right) \frac{\left(1-\alpha\left(\mu_{1}\right)\right) z_{2}}{1-\alpha\left(\mu_{1}\right) z_{2}}+\zeta}{1-\alpha\left(\mu_{2}\right) z_{2}} .
$$

Finally, Eqs. (56), (58), and (60) give the generating function of $\left(Y_{1}, Y_{2}\right)$ :

$$
\begin{aligned}
L\left(z_{1}, z_{2}\right)= & \frac{1}{1-\alpha\left(\mu_{1}+\mu_{2}\right) z_{1}} \\
& \times\left[\left(\alpha\left(\mu_{1}\right)-\alpha\left(\mu_{1}+\mu_{2}\right)\right) \frac{\left(1-\alpha\left(\mu_{1}\right)\right) z_{1}}{1-\alpha\left(\mu_{1}\right) z_{1}}\right.
\end{aligned}
$$




$$
\begin{aligned}
& +\left(\alpha\left(\mu_{2}\right)-\alpha\left(\mu_{1}+\mu_{2}\right)\right) \frac{z_{2}}{1-\alpha\left(\mu_{2}\right) z_{2}} \\
& \times\left(\zeta+\frac{\left(1-\alpha\left(\mu_{1}\right)\right) z_{2}}{1-\alpha\left(\mu_{1}\right) z_{2}}\left(\alpha\left(\mu_{1}\right)-\alpha\left(\mu_{1}+\mu_{2}\right)+\omega\right)\right) \\
& \left.+\omega \frac{\left(1-\alpha\left(\mu_{1}\right)\right) z_{2}}{1-\alpha\left(\mu_{1}\right) z_{2}}+\zeta\right] .
\end{aligned}
$$

Substituting $z_{2}=0$ in (61) gives

$$
L\left(z_{1}, 0\right)=\frac{\left(\alpha\left(\mu_{1}\right)-\alpha\left(\mu_{1}+\mu_{2}\right)\right) \frac{\left(1-\alpha\left(\mu_{1}\right)\right) z_{1}}{1-\alpha\left(\mu_{1}\right) z_{1}}+\zeta}{1-\alpha\left(\mu_{1}+\mu_{2}\right) z_{1}} .
$$

In a similar way we get $L\left(0, z_{2}\right)$ and $L\left(1, z_{2}\right)=E\left[z_{2}^{Y_{2}}\right]$. In particular,

$$
\begin{aligned}
L\left(1, z_{2}\right)= & \frac{1}{1-\alpha\left(\mu_{1}+\mu_{2}\right)} \\
& \times\left[\left(\alpha\left(\mu_{1}\right)-\alpha\left(\mu_{1}+\mu_{2}\right)\right)\right. \\
& +\left(\alpha\left(\mu_{2}\right)-\alpha\left(\mu_{1}+\mu_{2}\right)\right) \frac{z_{2}}{1-\alpha\left(\mu_{2}\right) z_{2}} \\
& \times\left(\zeta+\frac{\left(1-\alpha\left(\mu_{1}\right)\right) z_{2}}{1-\alpha\left(\mu_{1}\right) z_{2}}\left(\alpha\left(\mu_{1}\right)-\alpha\left(\mu_{1}+\mu_{2}\right)+\omega\right)\right) \\
& \left.+\omega \frac{\left(1-\alpha\left(\mu_{1}\right)\right) z_{2}}{1-\alpha\left(\mu_{1}\right) z_{2}}+\zeta\right] .
\end{aligned}
$$

Substituting $z_{2}=0$ in (61) gives

$$
L\left(z_{1}, 0\right)=\frac{\left(\alpha\left(\mu_{1}\right)-\alpha\left(\mu_{1}+\mu_{2}\right)\right) \frac{\left(1-\alpha\left(\mu_{1}\right)\right) z_{1}}{1-\alpha\left(\mu_{1}\right) z_{1}}+\zeta}{1-\alpha\left(\mu_{1}+\mu_{2}\right) z_{1}} .
$$

It is seen that the marginal distribution of $Y_{2}$ has an atom in 0 and furthermore is a weighted sum of (i) a geometric $\left(\alpha\left(\mu_{1}\right)\right)$ distribution, (ii) a geometric $\left(\alpha\left(\mu_{2}\right)\right)$ distribution, and (iii) a convolution of two such geometric distributions.

Finally, we determine the generating function of the joint distribution of the steadystate numbers of customers $\left(S_{1}, S_{2}\right)$ in $Q_{1}$ and $Q_{2}$ at an arbitrary epoch. It is easily seen that this distribution is obtained by considering the queue lengths at a time $A^{r}$ after the last customer arrival, where this forward recurrence interarrival time or residual interarrival time has LST $\alpha^{r}(s)=\frac{1-\alpha(s)}{s E[A]}$. We can follow the reasoning leading to (55), simply replacing each $\alpha(\cdot)$ term by $\alpha^{r}(\cdot)$. Hence

$$
\begin{aligned}
E\left[z_{1}^{S_{1}} z_{2}^{S_{2}}\right]= & \alpha^{r}\left(\mu_{1}+\mu_{2}\right) z_{1} L\left(z_{1}, z_{2}\right) \\
& +\left(\alpha^{r}\left(\mu_{1}\right)-\alpha^{r}\left(\mu_{1}+\mu_{2}\right)\right) z_{1} L\left(z_{1}, 1\right) \\
& +\left(\alpha^{r}\left(\mu_{2}\right)-\alpha^{r}\left(\mu_{1}+\mu_{2}\right)\right) z_{2} L\left(z_{2}, z_{2}\right) \\
& +\tilde{\omega} z_{2} L\left(z_{2}, 1\right)+\tilde{\zeta}
\end{aligned}
$$


where $\tilde{\omega}$ and $\tilde{\zeta}$ are obtained from $\omega$ and $\zeta$ by replacing $\alpha(\cdot)$ by $\alpha^{r}(\cdot)$ everywhere.

\section{Suggestions for further research}

The following extensions might be of interest:

1. Firstly, and perhaps most interestingly, there are various asymptotic questions. For example, one could let $n \rightarrow \infty$, and study, for example, the fraction of empty stations. We refer to Chapter 6 of [5] and to [7-9] for an interesting collection of limit laws for three limiting regimes (for the case of only arrivals at $Q_{1}$, and exponential gate openings): (i) The heavy-traffic regime, in which the arrival rate at $Q_{1}$ goes to infinity; (ii) the large-system regime in which $n \rightarrow \infty$; (iii) the balanced-system regime, in which $n \rightarrow \infty$, the gate opening intervals tend to zero, and the product of $n$ and the mean gate opening interval tends to a positive limit.

2. We are presently exploring ASIP models with finite waiting rooms. In such a case it is, for example, interesting to allocate the waiting room sizes - under a constraint on total waiting room size-such that the throughput of the ASIP is as large as possible.

3. A batch can move one or two queues ahead at a gate opening. The approach taken in Sect. 3 to obtain expressions for the $G_{k i}(z)$ (cf. (1)) breaks down when batches could move more than one queue ahead after a gate opening.

4. At each gate opening, multiple gates can open. If, with probability $r_{i}$, gates $i$ and $i+1$ open, $i=1,2, \ldots$, then this amounts to a batch moving two queues ahead. So this variant is related to the previous one.

5. Nontandem configurations. For example, there are three queues, $Q_{1}$ feeding into $Q_{2}$ and $Q_{3}$-with fixed probabilities, or via a fixed alternating pattern.

Acknowledgments Onno Boxma gratefully acknowledges interesting discussions with Corné Suijkerbuijk.

Open Access This article is distributed under the terms of the Creative Commons Attribution 4.0 International License (http://creativecommons.org/licenses/by/4.0/), which permits unrestricted use, distribution, and reproduction in any medium, provided you give appropriate credit to the original author(s) and the source, provide a link to the Creative Commons license, and indicate if changes were made.

\section{References}

1. Blythe, R.A., Evans, M.R.: Nonequilibrium steady states of matrix product form: a solver's guide. J. Phys. A Math. Theor. 40, R333-R441 (2007)

2. Derrida, B.: An exactly soluble non-equilibrium system: the asymmetric simple exclusion process. Phys. Rep. 301, 65-83 (1998)

3. Golinelli, O., Mallick, K.: The asymmetric simple exclusion process: an integrable model for nonequilibrium statistical mechanics. J. Phys. A Math. Gen. 39, 12679-12705 (2006)

4. Ibaraki, T.I., Katoh, N.: Resource Allocation Problems. MIT Press, Cambridge (1988)

5. Reuveni, S.: Tandem Stochastic Systems: The Asymmetric Simple Inclusion Process. PhD Thesis, Tel-Aviv University (2014)

6. Reuveni, S., Eliazar, I., Yechiali, U.: Asymmetric inclusion process. Phys. Rev. E 84(041101), 1-16 (2011) 
7. Reuveni, S., Eliazar, I., Yechiali, U.: Limit laws for the asymmetric inclusion process. Phys. Rev. E 86(061133), 1-17 (2012)

8. Reuveni, S., Eliazar, I., Yechiali, U.: Asymmetric inclusion process as a showcase of complexity. Phys. Rev. Lett. 109(020603), 1-4 (2012)

9. Reuveni, S., Hirschberg, O., Eliazar, I., Yechiali, U.: Occupation probabilities and fluctuations in the asymmetric inclusion process. Phys. Rev. E 89(042109), 1-23 (2014) 